\title{
SUPERHUMPS IN THE SU UMA STAR AK CNC
}

\author{
R. MENNICKENT ${ }^{1}$, D. NOGAMI ${ }^{2}$, T. KATO ${ }^{2}$, \\ W. WORRAKER ${ }^{3}$ \\ 1. Dpto. de Física, Instituto de Cs. Físicas y Matemáticas, \\ University de Concepción, Casilla 4009, Concepción, Chile \\ 2. Dept. Astron., Faculty of Sci., Kyoto University, \\ Oiwake-cho, Kitashirakawa, Saykyo-ku, Kyto 606-01, Japan \\ 3. British Astronomical Association, 65 Wantage Road, \\ Didcot, Oxon, OX11 OAE, UK
}

\begin{abstract}
We have observed AK Cnc during the 1995 March superoutburst, by means of differential $V-U$ photometry. The observations were obtained at Las Campanas (Chile), Ouda Station (Kyoto, Japan) and in England. Superhumps with amplitude $0.2 \mathrm{mag}$ appeared at least $4 \mathrm{~d}$ after maximum. They evolved from a single peak structure, developing a secondary peak during decline, until they reached a double-wave modulation with $0.05 \mathrm{mag}$ amplitude, after $8 \mathrm{~d}$. After removing linear trends in our data, the following ephemeris was found:

$$
U T_{\max }(1995 \mathrm{March})=12.152(1)+0.06749(1) N \mathrm{~d} .
$$

This superhump period was confirmed, independently, with our homogeneous set of $462 \mathrm{~V}$ magnitudes, obtained at LCO, then adding $63 \mathrm{U}$ data points and finally considering all the dataset, consisting of $789 \mathrm{~V}+U$ magnitudes. The superhump period is slightly larger than that reported by Kato (1995) during the 1992 January superoutburst $(0.06735(5) \mathrm{d})$. Our results imply an orbital period $\mathrm{P}_{\text {orb }}=0.065(2) \mathrm{d}$, using the relationship given by Howell \& Hurst (1994). The eruption duration was $\gtrsim 10 \mathrm{~d}$, and its amplitude $>6 \mathrm{mag}$. An abrupt and short luminosity decrease occured $\approx$ $12 \mathrm{~d}$ after maximum. Eleven outbursts have been observed since 1992 January, being the minimum reported lag between successive eruptions: $40 \mathrm{~d}$. More details will be given in a future publication.
\end{abstract}

\section{References}

Howell, S., Hurst, G., 1994, IBVS, 4043

Kato, T., 1995, IBVS, 4136 


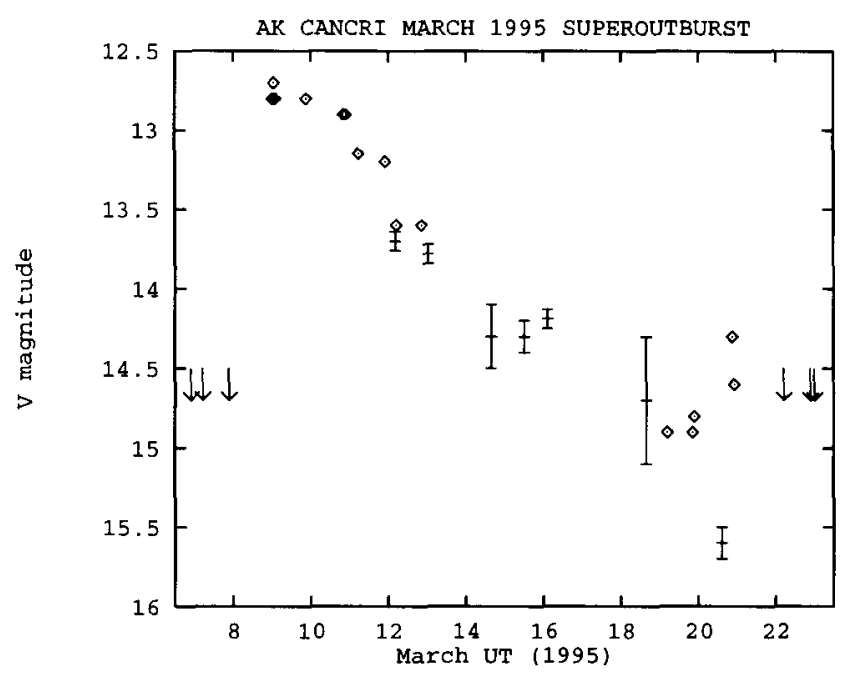

Figure 1. $\quad V$ light curve of AK Cnc during the 1995 March superoutburst. Data are from the British Astronomical Association Variable Star Section database $(\diamond)$ and from our own observations $(+)$. The reported quiescent magnitude is $V \approx 19 \mathrm{mag}$. Note the sudden brightness drop on March 20.

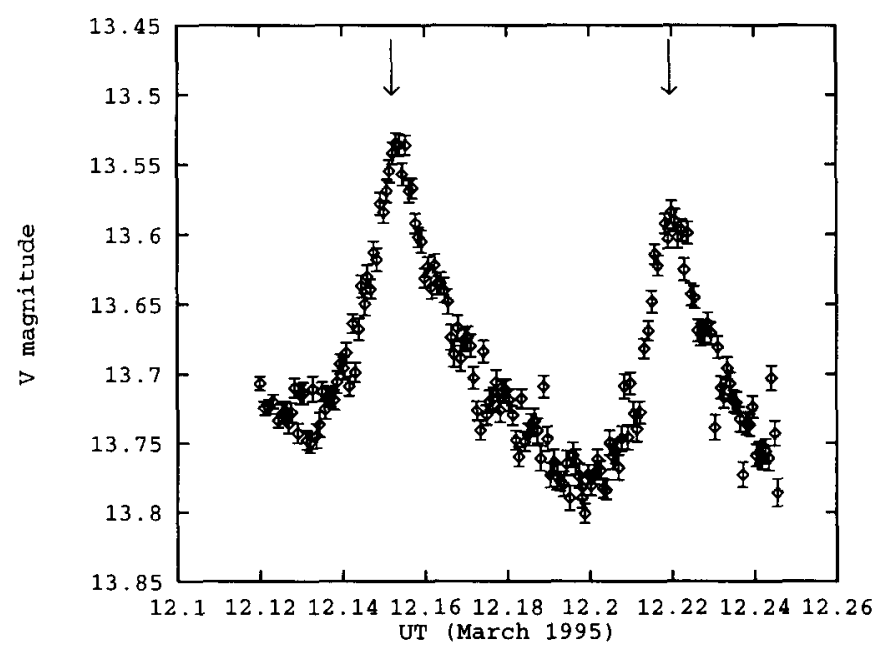

Figure 2. CCD $V$ light curve of AK Cnc. Arrows indicate times of maximum derived from equation (1). The star is fading at rate $0.4 \pm 0.1 \mathrm{mag} \mathrm{d}^{-1}$. Note the quasi-periodic signal during decline (time-scale $T \approx 13.3 \mathrm{~m}$ ). 